\title{
Multilepton Higgs decays through the dark portal
}

\author{
Chia-Feng Chang, ${ }^{a}$ Ernest $\mathrm{Ma}^{b}$ and Tzu-Chiang Yuan ${ }^{c}$ \\ ${ }^{a}$ Department of Physics, National Taiwan Normal University, \\ Taipei 116, Taiwan \\ ${ }^{b}$ Department of Physics and Astronomy, University of California, \\ Riverside, California 92521, U.S.A. \\ ${ }^{c}$ Institute of Physics, Academia Sinica, \\ Nangang, Taipei 11529, Taiwan \\ E-mail: 60141014S@ntnu.edu.tw, ma@phyun8.ucr.edu, \\ tcyuan@phys.sinica.edu.tw
}

ABstract: The $\mathrm{U}(1)_{D}$ gauge sector containing one dark Higgs boson $h_{D}$ and one dark photon $\gamma_{D}$ may be explored through the decays of the $126 \mathrm{GeV}$ particle discovered at the Large Hadron Collider (LHC), assumed here as the heavier mass eigenstate $h_{1}$ in the mixing of the standard model $h$ with $h_{D}$. The various decays of $h_{1}$ to $\gamma_{D} \gamma_{D}, h_{2} h_{2}, h_{2} \gamma_{D} \gamma_{D}$ and $h_{2} h_{2} h_{2}$ would yield multilepton final states through the mixing of $\gamma_{D}$ with the photon and the decay $h_{2} \rightarrow \gamma_{D} \gamma_{D}$, where $h_{2}$ is the lighter dark Higgs. Future searches for signals of multilepton jets at the LHC may reveal the existence of this possible dark sector governed simply by the original Abelian Higgs model.

KeYwords: Gauge Symmetry, Higgs Physics, Beyond Standard Model

ARXiv EPRINT: 1308.6071 


\section{Contents}

1 Introduction 1

$2 \mathrm{SU}(2)_{L} \times \mathrm{U}(1)_{Y} \times \mathrm{U}(1)_{D}$ model 2

3 Mixing effects 3

3.1 Higgs mass eigenstates and their self interactions 3

3.2 Kinetic and mass mixing of the neutral gauge bosons 4

4 Non-standard decays of $h_{1} \quad 6$

$\begin{array}{llr}5 & \text { Branching ratios } & 8\end{array}$

6 Multilepton jets at the LHC 11

$\begin{array}{lll}7 & \text { Conclusions } & 15\end{array}$

\section{Introduction}

The original Higgs model [1-3] of spontaneous symmetry breaking involves just one complex scalar field $\chi$ and one vector gauge field $C$. As $\chi$ acquires a nonzero vacuum expectation value $(\mathrm{VEV})$, the physical spectrum of this model consists of a massive vector boson $\gamma_{D}$ and a massive real scalar boson $h_{D}$, and the only interactions between them are of the form $h_{D} \gamma_{D} \gamma_{D}$ and $h_{D}^{2} \gamma_{D} \gamma_{D}$. The analog of $h_{D}$ in the electroweak $\mathrm{SU}(2) \times \mathrm{U}(1)$ extension [4] of this original model, commonly called the Higgs boson $h$, is presumably the $126 \mathrm{GeV}$ particle observed at the Large Hadron Collider (LHC) $[5,6]$. Is this the whole story? Perhaps not, because the original Higgs model may still be realized physically, but in a sector which connects with the standard model (SM) of particle interactions only through $h_{D}-h$ mass mixing and $\gamma_{D}-\gamma$ kinetic mixing $[7,8]$. If so, the $126 \mathrm{GeV}$ particle may be identified with the heavier mass eigenstate $h_{1}$ and decays such as $h_{1}$ to $\gamma_{D} \gamma_{D}, h_{2} h_{2}, h_{2} \gamma_{D} \gamma_{D}$ and $h_{2} h_{2} h_{2}$ would result in multilepton final states via $\gamma_{D} \rightarrow \bar{l} l$ or $h_{2} \rightarrow \gamma_{D} \gamma_{D}$ and then followed by $\gamma_{D} \rightarrow \overline{l l}$, where $h_{2}$ is the lighter dark Higgs and $l$ is the SM lepton.

In section 2 we set up our model. Phenomenology based on similar model has been studied before, see for example refs. [9-14] and references therein. In section 3 we consider mixing effects in the scalar sector as well as the gauge boson sector. We show the $h_{D}-h$ mixing in detail and present all the relevant trilinear and quadrilinear couplings of the physical $h_{1}$ and $h_{2}$ bosons. We also briefly discuss the mixings between the three neutral gauge bosons in the model as studied previously in ref. [14]. In section 4 we discuss the possible decay modes of the SM Higgs outside those of the SM and their several kinematic 
regions. In section 5 we present numerical results for various branching ratios of the nonstandard decay modes of the SM Higgs, identified here as $h_{1}$. In section 6 we study the signals of multilepton jets of the model at the LHC-14. We conclude in section 7 .

\section{$2 \mathrm{SU}(2)_{L} \times \mathrm{U}(1)_{Y} \times \mathrm{U}(1)_{D}$ model}

We extend the electroweak SM by including the original Abelian Higgs model for a dark $\mathrm{U}(1)_{D}[9-14]$. The bosonic part of the Lagrangian density is

$$
\mathcal{L}_{\mathrm{B}}=\mathcal{L}_{\text {gauge }}+\mathcal{L}_{\text {scalar }}
$$

with

$$
\begin{aligned}
& \mathcal{L}_{\text {gauge }}=-\frac{1}{4} \vec{W}_{\mu \nu} \cdot \vec{W}^{\mu \nu}-\frac{1}{4} B_{\mu \nu} B^{\mu \nu}-\frac{1}{4} C_{\mu \nu} C^{\mu \nu}-\frac{\epsilon}{2} B_{\mu \nu} C^{\mu \nu} \\
& \mathcal{L}_{\text {scalar }}=\left|D_{\mu} \Phi\right|^{2}+\left|D_{\mu} \chi\right|^{2}-V_{\text {scalar }}(\Phi, \chi)
\end{aligned}
$$

and

$$
\begin{aligned}
D_{\mu} \Phi & =\left(\partial_{\mu}+i g \frac{1}{2} \sigma_{a} W_{a \mu}+i \frac{1}{2} g^{\prime} B_{\mu}\right) \Phi \\
D_{\mu} \chi & =\left(\partial_{\mu}+i g_{D} C_{\mu}\right) \chi
\end{aligned}
$$

where $\vec{W}^{\mu}, B^{\mu}$ and $C^{\mu}$ are the gauge potentials of the $\mathrm{SU}(2)_{L}, \mathrm{U}(1)_{Y}$ and $\mathrm{U}(1)_{D}$ with gauge couplings $g, g^{\prime}$ and $g_{D}$ respectively, and $\epsilon$ is the kinetic mixing parameter between the two $\mathrm{U}(1) \mathrm{s}[7,8]$. The scalar potential in $(2.3)$ is given by

$$
V_{\text {scalar }}=-\mu_{\Phi}^{2} \Phi^{\dagger} \Phi+\lambda_{\Phi}\left(\Phi^{\dagger} \Phi\right)^{2}-\mu_{\chi}^{2} \chi^{*} \chi+\lambda_{\chi}\left(\chi^{*} \chi\right)^{2}+\lambda_{\Phi \chi}\left(\Phi^{\dagger} \Phi\right)\left(\chi^{*} \chi\right)
$$

We pick the unitary gauge and expand the scalar fields around the vacuum

$$
\Phi(x)=\frac{1}{\sqrt{2}}\left(\begin{array}{c}
0 \\
v+h(x)
\end{array}\right) \quad, \quad \chi(x)=\frac{1}{\sqrt{2}}\left(v_{D}+h_{D}(x)\right)
$$

with the VEVs $v$ and $v_{D}$ fixed by minimisation of the potential to be

$$
v^{2}=\frac{\mu_{\Phi}^{2}-\frac{1}{2} \frac{\lambda_{\Phi \chi}}{\lambda_{\chi}} \mu_{\chi}^{2}}{\lambda_{\Phi}-\frac{1}{4} \frac{\lambda_{\Phi \chi}^{2}}{\lambda_{\chi}}}, \quad v_{D}^{2}=\frac{\mu_{\chi}^{2}-\frac{1}{2} \frac{\lambda_{\Phi \chi}}{\lambda_{\Phi}} \mu_{\Phi}^{2}}{\lambda_{\chi}-\frac{1}{4} \frac{\lambda_{\Phi \chi}^{2}}{\lambda_{\Phi}}} .
$$

In terms of the shifted fields $h$ and $h_{D}$, the scalar potential $V_{\text {scalar }}$ can then be decomposed as

$$
V_{\text {scalar }}=V_{0}+V_{1}+V_{2}+V_{3}+V_{4}
$$


with

$$
\begin{aligned}
V_{0} & =\frac{1}{4}\left(\lambda_{\Phi} v^{4}+\lambda_{\chi} v_{D}^{4}+\lambda_{\Phi \chi} v^{2} v_{D}^{2}-2 \mu_{\Phi}^{2} v^{2}-2 \mu_{\chi}^{2} v_{D}^{2}\right) \\
V_{1} & =\frac{1}{2} v\left(2 \lambda_{\Phi} v^{2}+\lambda_{\Phi \chi} v_{D}^{2}-2 \mu_{\Phi}^{2}\right) h+\frac{1}{2} v_{D}\left(2 \lambda_{\chi} v_{D}^{2}+\lambda_{\Phi \chi} v^{2}-2 \mu_{\chi}^{2}\right) h_{D} \\
V_{2} & =\left(\frac{3}{2} \lambda_{\Phi} v^{2}+\frac{1}{4} \lambda_{\Phi \chi} v_{D}^{2}-\frac{1}{2} \mu_{\Phi}^{2}\right) h^{2}+\left(\frac{3}{2} \lambda_{\chi} v_{D}^{2}+\frac{1}{4} \lambda_{\Phi \chi} v^{2}-\frac{1}{2} \mu_{\chi}^{2}\right) h_{D}^{2}+\lambda_{\Phi \chi} v v_{D} h h_{D} \\
& \equiv \frac{1}{2}\left(h h_{D}\right) \cdot M_{S}^{2} \cdot\left(\begin{array}{c}
h \\
h_{D}
\end{array}\right) \\
V_{3} & =\lambda_{\Phi} v h^{3}+\lambda_{\chi} v_{D} h_{D}^{3}+\frac{1}{2} \lambda_{\Phi \chi}\left(v_{D} h_{D} h^{2}+v h h_{D}^{2}\right) \\
V_{4} & =\frac{1}{4} \lambda_{\Phi} h^{4}+\frac{1}{4} \lambda_{\chi} h_{D}^{4}+\frac{1}{4} \lambda_{\Phi \chi} h^{2} h_{D}^{2}
\end{aligned}
$$

Here $V_{0}$ is a cosmological constant and will be discarded from now on; the tadpole term $V_{1}$ vanishes with $v$ and $v_{D}$ given by eq. (2.8); $V_{2}$ is quadratic in the fields $h$ and $h_{D}$, and we have to diagonalize the mass matrix $M_{S}^{2}$ in eq. (2.11) to get the physical Higgs fields $h_{1}$ and $h_{2}$ (see next section); and $V_{3}$ and $V_{4}$ are the trilinear and quadrilinear self couplings among the two Higgs fields. Since $\chi$ is a SM singlet, the $W$ and $Z$ bosons acquire their masses through the SM Higgs doublet VEV $v$ entirely which implies $v=246 \mathrm{GeV}$.

\section{$3 \quad$ Mixing effects}

\subsection{Higgs mass eigenstates and their self interactions}

The mass matrix $M_{S}^{2}$ in eq. (2.11) for the scalar bosons is

$$
\begin{aligned}
M_{S}^{2} & =\left(\begin{array}{ll}
m_{11}^{2} & m_{12}^{2} \\
m_{21}^{2} & m_{22}^{2}
\end{array}\right), \\
& =\left(\begin{array}{cc}
2 \lambda_{\Phi} v^{2} & \lambda_{\Phi \chi} v v_{D} \\
\lambda_{\Phi \chi} v v_{D} & 2 \lambda_{\chi} v_{D}^{2}
\end{array}\right) .
\end{aligned}
$$

Its eigenvalues are

$$
m_{1,2}^{2}=\frac{1}{2}\left[\operatorname{Tr} M_{S}^{2} \pm \sqrt{\left(\operatorname{Tr} M_{S}^{2}\right)^{2}-4 \operatorname{Det} M_{S}^{2}}\right] .
$$

The physical Higgs $\left(h_{1}, h_{2}\right)$ are related to the original $\left(h, h_{D}\right)$ as

$$
\left(\begin{array}{c}
h_{1} \\
h_{2}
\end{array}\right)=\left(\begin{array}{cc}
\cos \alpha & \sin \alpha \\
-\sin \alpha & \cos \alpha
\end{array}\right)\left(\begin{array}{c}
h \\
h_{D}
\end{array}\right),
$$

with the mixing angle

$$
\sin 2 \alpha=\frac{2 m_{12}^{2}}{m_{1}^{2}-m_{2}^{2}}
$$

We will identify the heavier Higgs $h_{1}$ with mass $m_{1}=126 \mathrm{GeV}$ as the new boson observed at the LHC $[5,6]$, while the lighter one $h_{2}$ has been escaped detection thus far. The SM 
Higgs couplings with the SM fermions and gauge bosons are thus modified by a factor of $\cos \alpha$.

In terms of the physical Higgs fields $h_{1}$ and $h_{2}$, the cubic term $V_{3}$ is given by

$$
V_{3}=\frac{1}{3 !} \lambda_{3}^{(1)} h_{1}^{3}+\frac{1}{3 !} \lambda_{3}^{(2)} h_{2}^{3}+\frac{1}{2} \lambda_{3}^{(3)} h_{1} h_{2}^{2}+\frac{1}{2} \lambda_{3}^{(4)} h_{2} h_{1}^{2}
$$

with the trilinear couplings

$$
\begin{gathered}
\lambda_{3}^{(1)}=3\left[2 v \lambda_{\Phi} \cos ^{3} \alpha+2 v_{D} \lambda_{\chi} \sin ^{3} \alpha+\frac{1}{2} \lambda_{\Phi \chi} \sin 2 \alpha\left(v \sin \alpha+v_{D} \cos \alpha\right)\right], \\
\lambda_{3}^{(2)}=3\left[-2 v \lambda_{\Phi} \sin ^{3} \alpha+2 v_{D} \lambda_{\chi} \cos ^{3} \alpha+\frac{1}{2} \lambda_{\Phi \chi} \sin 2 \alpha\left(v_{D} \sin \alpha-v \cos \alpha\right)\right], \\
\lambda_{3}^{(3)}=\frac{1}{4}\left[24 v \lambda_{\Phi} \sin ^{2} \alpha \cos \alpha+24 v_{D} \lambda_{\chi} \cos ^{2} \alpha \sin \alpha\right. \\
\left.\quad+\lambda_{\Phi \chi}\left(v \cos \alpha+v_{D} \sin \alpha+3 v \cos 3 \alpha-3 v_{D} \sin 3 \alpha\right)\right], \\
\lambda_{3}^{(4)}=\frac{1}{4}\left[-24 v \lambda_{\Phi} \sin \alpha \cos ^{2} \alpha+24 v_{D} \lambda_{\chi} \cos \alpha \sin ^{2} \alpha\right. \\
\left.\quad+\lambda_{\Phi \chi}\left(-v \sin \alpha+v_{D} \cos \alpha+3 v \sin 3 \alpha+3 v_{D} \cos 3 \alpha\right)\right]
\end{gathered}
$$

and the quartic term $V_{4}$ is given by

$$
V_{4}=\frac{1}{4 !} \lambda_{4}^{(1)} h_{1}^{4}+\frac{1}{4 !} \lambda_{4}^{(2)} h_{2}^{4}+\frac{1}{3 !} \lambda_{4}^{(3)} h_{1} h_{2}^{3}+\frac{1}{3 !} \lambda_{4}^{(4)} h_{2} h_{1}^{3}+\frac{1}{2 ! \cdot 2 !} \lambda_{4}^{(5)} h_{1}^{2} h_{2}^{2}
$$

with the quadrilinear couplings

$$
\begin{aligned}
& \lambda_{4}^{(1)}=6\left(\lambda_{\Phi} \cos ^{4} \alpha+\lambda_{\chi} \sin ^{4} \alpha+\frac{1}{4} \lambda_{\Phi \chi} \sin ^{2} 2 \alpha\right), \\
& \lambda_{4}^{(2)}=6\left(\lambda_{\Phi} \sin ^{4} \alpha+\lambda_{\chi} \cos ^{4} \alpha+\frac{1}{4} \lambda_{\Phi \chi} \sin ^{2} 2 \alpha\right), \\
& \lambda_{4}^{(3)}=-\frac{3}{2} \sin 2 \alpha\left(-2 \lambda_{\chi} \cos ^{2} \alpha+2 \lambda_{\Phi} \sin ^{2} \alpha+\lambda_{\Phi \chi} \cos 2 \alpha\right), \\
& \lambda_{4}^{(4)}=+\frac{3}{2} \sin 2 \alpha\left(2 \lambda_{\chi} \sin ^{2} \alpha-2 \lambda_{\Phi} \cos ^{2} \alpha+\lambda_{\Phi \chi} \cos 2 \alpha\right), \\
& \lambda_{4}^{(5)}=\frac{1}{4}\left[3\left(\lambda_{\Phi}+\lambda_{\chi}\right)+\lambda_{\Phi \chi}-3\left(\lambda_{\Phi}+\lambda_{\chi}-\lambda_{\Phi \chi}\right) \cos 4 \alpha\right] .
\end{aligned}
$$

\subsection{Kinetic and mass mixing of the neutral gauge bosons}

In additional to the mass mixing of the three neutral gauge bosons arise from the spontaneously electroweak symmetry breaking given by

$$
\mathcal{L}_{\mathrm{m}}=\frac{1}{2}\left(C^{\mu} B^{\mu} W^{3 \mu}\right) M^{2}\left(\begin{array}{c}
C_{\mu} \\
B_{\mu} \\
W_{\mu}^{3}
\end{array}\right)
$$

with the following mass matrix

$$
M^{2}=\left(\begin{array}{ccc}
g_{D}^{2} v_{D}^{2} & 0 & 0 \\
0 & \frac{1}{4} g^{\prime 2} v^{2} & -\frac{1}{4} g g^{\prime} v^{2} \\
0 & -\frac{1}{4} g g^{\prime} v^{2} & \frac{1}{4} g^{2} v^{2}
\end{array}\right),
$$


we also have the kinetic mixing between the two U(1)s from the last term in eq. (2.2). Both the kinetic and mass mixings can be diagonalized simultaneously by the following mixed transformation $[14]$

$$
\left(\begin{array}{c}
C_{\mu} \\
B_{\mu} \\
W_{\mu}^{3}
\end{array}\right)=K \cdot O\left(\begin{array}{c}
A_{\mu}^{\prime} \\
Z_{\mu} \\
A_{\mu}
\end{array}\right)
$$

where $A_{\mu}^{\prime}, Z_{\mu}$ and $A_{\mu}$ are the physical dark photon, $Z$ boson and the photon respectively. Here $K$ is a general linear transformation that diagonalizes the kinetic mixing

$$
K=\left(\begin{array}{ccc}
\beta & 0 & 0 \\
-\epsilon \beta & 1 & 0 \\
0 & 0 & 1
\end{array}\right)
$$

where $\beta=1 /\left(1-\epsilon^{2}\right)^{1 / 2}(\epsilon \leq 0)$, and $O$ is a $3 \times 3$ orthogonal matrix which can be parametrized as

$$
O=\left(\begin{array}{ccc}
\cos \psi \cos \phi-\sin \theta \sin \phi \sin \psi & \sin \psi \cos \phi+\sin \theta \sin \phi \cos \psi & -\cos \theta \sin \phi \\
\cos \psi \sin \phi+\sin \theta \cos \phi \sin \psi & \sin \psi \sin \phi-\sin \theta \cos \phi \cos \psi & \cos \theta \cos \phi \\
-\cos \theta \sin \psi & \cos \theta \cos \psi & \sin \theta
\end{array}\right)
$$

with the mixing angles given by [14]

$$
\tan \theta=\frac{g^{\prime}}{g}, \quad \tan \phi=-\epsilon \beta, \quad \tan \psi= \pm \frac{\tan \phi \cos \theta}{\tan \theta}\left[\frac{1-M_{Z}^{2} / M_{W}^{2}}{1-M_{Z}^{2} / g_{D}^{2} v_{D}^{2}}+\tan ^{2} \theta\right] .
$$

After the $K$ transformation, the gauge boson mass matrix is

$$
\tilde{M}^{2}=K^{T} M^{2} K=\left(\begin{array}{ccc}
\beta^{2}\left(g_{D}^{2} v_{D}^{2}+\frac{1}{4} \epsilon^{2} g^{\prime 2} v^{2}\right) & -\frac{1}{4} \epsilon \beta g^{\prime 2} v^{2} & \frac{1}{4} \epsilon \beta g g^{\prime} v^{2} \\
-\frac{1}{4} \epsilon \beta g^{\prime 2} v^{2} & \frac{1}{4} g^{\prime 2} v^{2} & -\frac{1}{4} g g^{\prime} v^{2} \\
\frac{1}{4} \epsilon \beta g g^{\prime} v^{2} & -\frac{1}{4} g g^{\prime} v^{2} & \frac{1}{4} g^{2} v^{2}
\end{array}\right) .
$$

The $O$ matrix diagonalizes this $\tilde{M}^{2}$ matrix

$$
M_{\text {Diag }}^{2}=O^{T} \tilde{M}^{2} O=\left(\begin{array}{ccc}
M_{\gamma_{D}}^{2} & 0 & 0 \\
0 & M_{Z}^{2} & 0 \\
0 & 0 & M_{\gamma}^{2}
\end{array}\right)
$$

with the following eigenvalues (assuming $\left.M_{\gamma_{D}} \leq M_{Z}\right)^{1}$

$$
M_{\gamma}^{2}=0, \quad M_{Z, \gamma_{D}}^{2}=(q \pm p) / 2
$$

where

$$
\begin{aligned}
& p=\sqrt{q^{2}-g_{D}^{2} v_{D}^{2} v^{2}\left(g^{2}+g^{\prime 2}\right) \beta^{2}}, \\
& q=g_{D}^{2} v_{D}^{2} \beta^{2}+\frac{1}{4}\left(g^{2}+g^{\prime 2} \beta^{2}\right) v^{2} .
\end{aligned}
$$

\footnotetext{
${ }^{1}$ For the case of $M_{\gamma_{D}}>M_{Z}$, we will have $M_{\gamma_{D}, Z}^{2}=(q \pm p) / 2$ as was studied by the authors in [14].
} 
For small kinetic parameter mixing $\epsilon$, the $Z$ and $\gamma_{D}$ masses can be approximated by $M_{Z} \approx \sqrt{\left(g^{2}+g^{\prime 2}\right)} v / 2$ and $M_{\gamma_{D}} \approx g_{D} v_{D}$.

For couplings of these physical neutral gauge bosons with the SM fermions, we refer the readers to ref. [14].

\section{Non-standard decays of $h_{1}$}

The global fits [15-18] for the signal strengths of the various SM Higgs decay channels from the LHC data imply the total width of the SM Higgs is about $4.03 \mathrm{MeV}$ and the non-standard width for the SM Higgs can be at most $1.2 \mathrm{MeV}$; in other words the nonstandard branching ratio for the SM Higgs must be less than $22 \%$. One can use this result to constrain the parameter space of the model.

We will compute the following non-standard processes $h_{1} \rightarrow \gamma_{D} \gamma_{D}, h_{1} \rightarrow h_{2} h_{2}, h_{1} \rightarrow$ $h_{2} h_{2}^{*} \rightarrow h_{2} \gamma_{D} \gamma_{D}$ and $h_{1} \rightarrow h_{2} h_{2} h_{2}$. Each of the $h_{2}$ in the final state of these processes will decay into two dark photons and each dark photon will give rise to two leptons through its mixing with the photon. ${ }^{2}$ These non-standard processes will provide multiple leptons in the final state of the standard model Higgs decay [12]. The contribution to the heavier Higgs width from these non-standard processes is ${ }^{3}$

$$
\Gamma_{h_{1}}^{\mathrm{NS}}=\sin ^{2} \alpha \hat{\Gamma}\left(h_{1} \rightarrow \gamma_{D} \gamma_{D}\right)+\Gamma\left(h_{1} \rightarrow h_{2} h_{2}\right)+\Gamma\left(h_{1} \rightarrow h_{2} \gamma_{D} \gamma_{D}\right)+\Gamma\left(h_{1} \rightarrow h_{2} h_{2} h_{2}\right)+\cdots
$$

Thus the total width of the heavier Higgs $h_{1}$ is modified as

$$
\Gamma_{h_{1}}=\cos ^{2} \alpha \hat{\Gamma}_{h}+\Gamma_{h_{1}}^{\mathrm{NS}}
$$

where $\hat{\Gamma}_{h}$ is the width of the SM Higgs $h$, which has a theoretical value of $4.03 \mathrm{MeV}$. The branching ratio for the non-standard modes of the heavier Higgs decay is

$$
B_{h_{1}}^{\mathrm{NS}}=\frac{\Gamma_{h_{1}}^{\mathrm{NS}}}{\Gamma_{h_{1}}},
$$

which should be constrained to be less than $22 \%$ or so. The partial decay width for the two body decays are given by

$$
\hat{\Gamma}\left(h_{1} \rightarrow \gamma_{D} \gamma_{D}\right)=\frac{g_{D}^{2} m_{\gamma_{D}}^{2}}{8 \pi m_{1}}\left(1-\frac{4 m_{\gamma_{D}}^{2}}{m_{1}^{2}}\right)^{\frac{1}{2}}\left(3-\frac{m_{1}^{2}}{m_{\gamma_{D}}^{2}}+\frac{m_{1}^{4}}{4 m_{\gamma_{D}}^{4}}\right),
$$

and

$$
\Gamma\left(h_{1} \rightarrow h_{2} h_{2}\right)=\frac{\left(\lambda_{3}^{(3)}\right)^{2}}{32 \pi m_{1}}\left(1-\frac{4 m_{2}^{2}}{m_{1}^{2}}\right)^{\frac{1}{2}} .
$$

\footnotetext{
${ }^{2}$ We note that $h_{2}$ can decay to SM particles as well through its mixing with $h_{1}$ and hence they are suppressed. We take the branching ratio of $h_{2} \rightarrow \gamma_{D} \gamma_{D}$ to be $100 \%$. See discussion after eq. (4.11).

${ }^{3}$ The four lepton modes from the first term $h_{1} \rightarrow \gamma_{D} \gamma_{D}$ followed by $\gamma_{D} \rightarrow l \bar{l}(l=e, \mu)$ were studied in details in [9].
} 
For the three body decay $h_{1} \rightarrow h_{2} h_{2} h_{2}$, we obtain

$$
\Gamma\left(h_{1} \rightarrow h_{2} h_{2} h_{2}\right)=\int_{x_{1}^{\min }}^{x_{1}^{\max }} d x_{1} \int_{x_{2}^{\min }}^{x_{2}^{\max }} d x_{2} \frac{d \Gamma\left(h_{1} \rightarrow h_{2} h_{2} h_{2}\right)}{d x_{1} d x_{2}}
$$

with the following differential decay rate

$$
\frac{d \Gamma\left(h_{1} \rightarrow h_{2} h_{2} h_{2}\right)}{d x_{1} d x_{2}}=\frac{m_{1}}{1536 \pi^{3}}|\mathcal{M}|^{2}
$$

where the matrix element is given by

$$
\mathcal{M}=\lambda_{4}^{(3)}+\frac{1}{m_{1}^{2}}\left(\lambda_{3}^{(2)} \lambda_{3}^{(3)} \sum_{i=1,2,3}\left(1-x_{i}\right)^{-1}+\lambda_{3}^{(3)} \lambda_{3}^{(4)} \sum_{i=1,2,3}\left(\mu-x_{i}\right)^{-1}\right),
$$

with $\mu=m_{2}^{2} / m_{1}^{2}$ and $x_{1}+x_{2}+x_{3}=2$. The range of integration for $x_{1}$ and $x_{2}$ is confined by

$$
\begin{gathered}
2 \sqrt{\mu}<x_{1}<1-3 \mu \\
x_{2}>\frac{1}{2}\left(1+\mu-x_{1}\right)^{-1}\left[\left(2-x_{1}\right)\left(1+\mu-x_{1}\right) \pm\left(x_{1}^{2}-4 \mu\right)^{1 / 2} \lambda^{1 / 2}\left(1+\mu-x_{1}, \mu, \mu\right)\right]
\end{gathered}
$$

where $\lambda(a, b, c)=a^{2}+b^{2}+c^{2}-2(a b+b c+c a)$.

The matrix element for the three body process $h_{1} \rightarrow h_{2} \gamma_{D} \gamma_{D}$ is rather long, we will not present the expression here but it is included entirely in our numerical work.

Now the dark Higgs $h_{2}$ decays into $\gamma_{D} \gamma_{D}$ and SM particles with coefficients $\cos ^{2} \alpha$ and $\sin ^{2} \alpha$ respectively, so its branching fraction into $\gamma_{D} \gamma_{D}$ is given by

$$
B\left(h_{2} \rightarrow \gamma_{D} \gamma_{D}\right)=\frac{\cos ^{2} \alpha \hat{\Gamma}\left(h_{2} \rightarrow \gamma_{D} \gamma_{D}\right)}{\cos ^{2} \alpha \hat{\Gamma}\left(h_{2} \rightarrow \gamma_{D} \gamma_{D}\right)+\sin ^{2} \alpha \hat{\Gamma}_{h_{2}}^{\mathrm{SM}}},
$$

where $\hat{\Gamma}\left(h_{2} \rightarrow \gamma_{D} \gamma_{D}\right)$ can be obtained from eq. (4.4) with the following substitution $m_{1} \rightarrow$ $m_{2}$, and $\hat{\Gamma}_{h_{2}}^{\mathrm{SM}}$ is the partial decay width of $h_{2}$ into SM particles. Since $\hat{\Gamma}_{h_{2}}^{\mathrm{SM}}$ are suppressed by a factor of $\sin ^{2} \alpha$, the above branching fraction is close to unity.

In figure 1 , the various regions of kinematics in the $\left(m_{\gamma_{D}}, m_{2}\right)$ plane that exhibits the very rich Higgs phenomenology in this model are schematically shown. The different regions can be described briefly as follows:

- Clearly, the two lines $2 m_{\gamma_{D}}=m_{1}$ (left of which $h_{1} \rightarrow \gamma_{D} \gamma_{D}$ is open) and $2 m_{2}=m_{1}$ (below of which $h_{1} \rightarrow h_{2} h_{2}$ is open) defines our region of interest (un-shaded).

- Below the line $3 m_{2}=m_{1}$, the 3 -body process $h_{1} \rightarrow h_{2} h_{2} h_{2}$ is open too.

- Other lines correspond to 2, 4, or 6 dark photons coming from the decays of $h_{2}$ in $h_{1} \rightarrow h_{2} h_{2}$ or $h_{1} \rightarrow h_{2} h_{2} h_{2}$ : i.e. to the left of the 5 lines $2 m_{\gamma_{D}}+m_{2}=m_{1}$, $2 m_{\gamma_{D}}+2 m_{2}=m_{1}, 4 m_{\gamma_{D}}+m_{2}=m_{1}, 4 m_{\gamma_{D}}=m_{1}$ and $6 m_{\gamma_{D}}=m_{1}$ correspond to the openings of the 5 processes $h_{1} \rightarrow h_{2} \gamma_{D} \gamma_{D}, h_{1} \rightarrow h_{2} h_{2} \gamma_{D} \gamma_{D}, h_{1} \rightarrow h_{2} \gamma_{D} \gamma_{D} \gamma_{D} \gamma_{D}$, $h_{1} \rightarrow \gamma_{D} \gamma_{D} \gamma_{D} \gamma_{D}$ and $h_{1} \rightarrow \gamma_{D} \gamma_{D} \gamma_{D} \gamma_{D} \gamma_{D} \gamma_{D}$ respectively. 


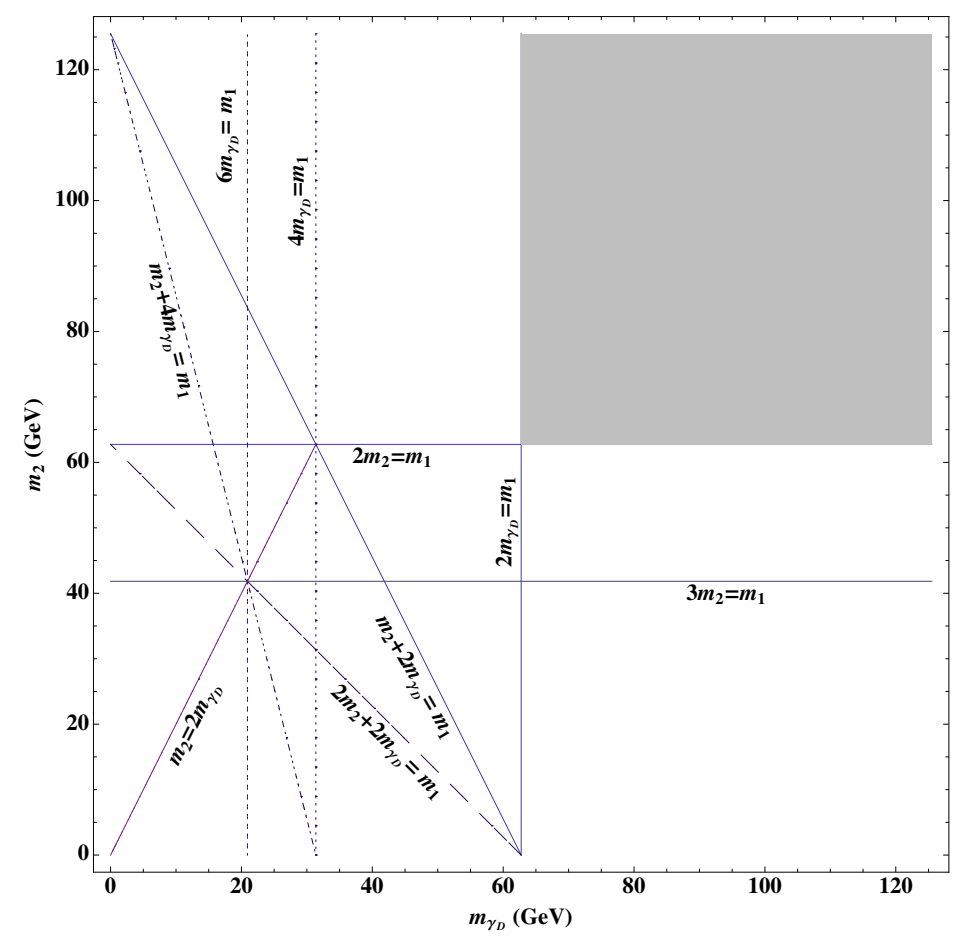

Figure 1. The kinematical regions in the $\left(m_{\gamma_{D}}, m_{2}\right)$ plane for the non-standard decays of the heavier Higgs $h_{1}$, identified as the $126 \mathrm{GeV}$ boson observed at LHC. See the last paragraph of section IV for illustrations.

- Lastly, the special line $m_{2}=2 m_{\gamma_{D}}$ emanated from the coordinate origin separates the $\gamma_{D} \gamma_{D}$ pair coming from either a on-shell $h_{2}$ or off-shell $h_{2}^{*}$ for these multi- $\gamma_{D}$ processes.

Since the dark photon $\gamma_{D}$ will mix with the photon, through either kinetic mixing $[7,8]$ or a gauge invariant Stueckelberg mass term [19, 20], it will communicate with the SM fermions eventually. If the dark photon mass is larger than twice the electron or muon mass, theses processes will lead to multileptons in the final states of the $h_{1}$ decay. These lepton jets can be distinguished from the QCD jets by imposing cuts on the electromagnetic ratio and charge ratio, as proposed in [12]. Supersymmetric models with or without Rparity can also give rise to multilepton events as experimentalists had searched for such signals and placed exclusion limits on the masses of supersymmetric particles [21]. LHC search for multilepton Higgs decay modes in the dark portal model will be discussed later in section 6 .

\section{Branching ratios}

In our numerical work, we will restrict our interest where both the dark photon and dark Higgs have masses smaller than $126 \mathrm{GeV}$. In particular, we will pay special attention to the small mass region where their masses are in the range of 0.5 to a few $\mathrm{GeV}$. In this range, final states of $\tau$ pair and light quarks pairs (pion and kaon pairs) from the dark photon decay are also possible, but they are harder to detect at the LHC. 

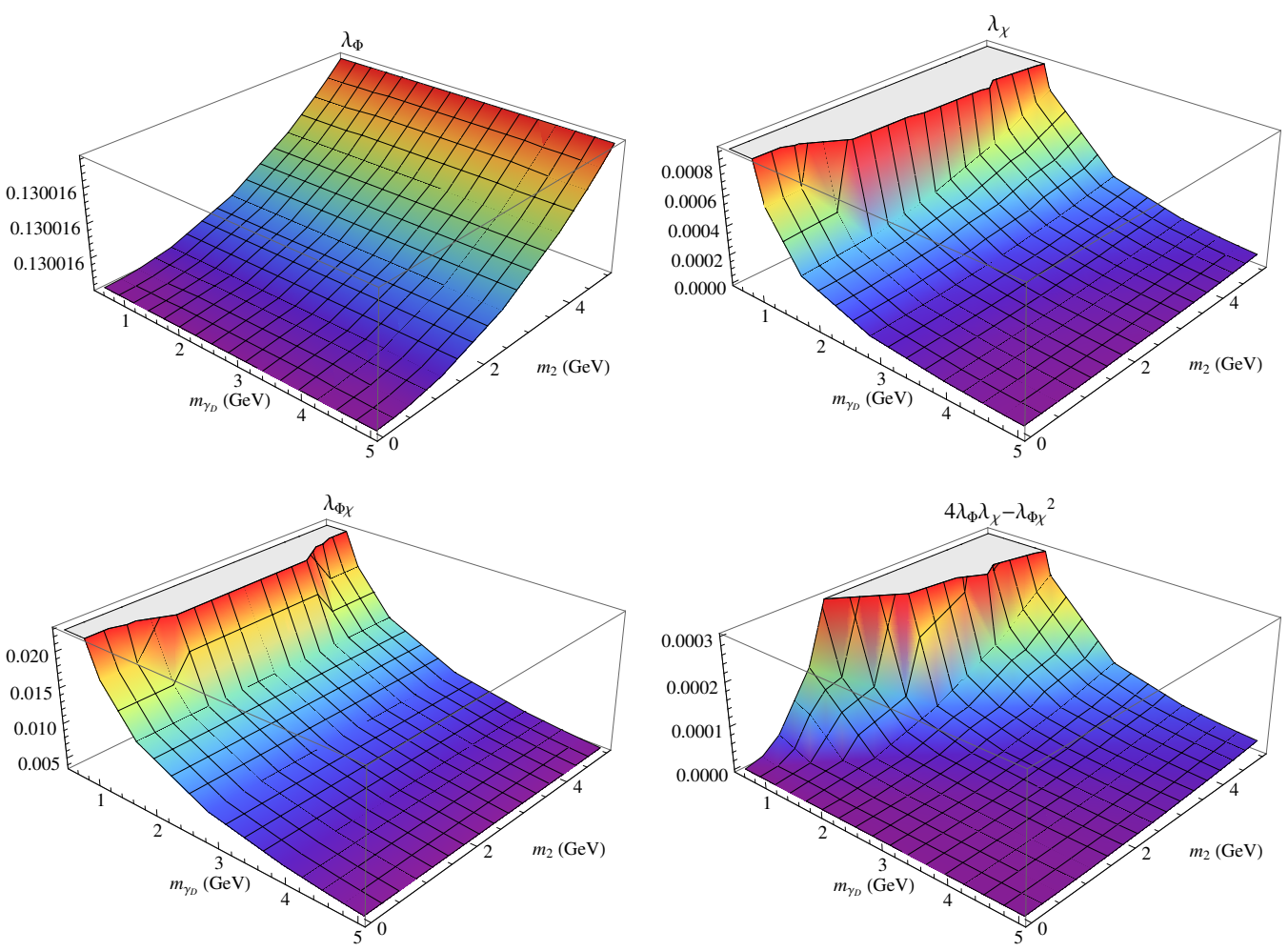

Figure 2. Fundamental couplings $\lambda_{\Phi}, \lambda_{\chi}$ and $\lambda_{\Phi \chi}$, and their combination $\left(4 \lambda_{\Phi} \lambda_{\chi}-\lambda_{\Phi \chi}^{2}\right)$ as function of $\left(m_{\gamma_{D}}, m_{2}\right)$ in the small mass region up to $5 \mathrm{GeV}$ with fixed values of $g_{D}=0.01$ and $\alpha=0.03$.

Limit for invisibly decay of a Higgs boson with mass as low as $1 \mathrm{GeV}$ had been reported by OPAL [22]. ${ }^{4}$ For a $1 \mathrm{GeV}$ Higgs boson mass, an upper limit for the mixing angle of $|\alpha| \leq 3 \times 10^{-2}$ can be extracted from the figure 5 in ref. [22]. However the exclusion curve on the Higgs mass versus mixing angle plot given in [22] was obtained under the assumption that invisible branching ratio of the Higgs boson decay is $100 \%$. Relaxing this assumption would lead to larger mixing angle for a given Higgs mass. In the present case, the branching ratio in eq. (4.3) must be less than $20 \%$ or so.

In figure 2, we plot the fundamental couplings $\lambda_{\Phi}, \lambda_{\chi}$ and $\lambda_{\Phi \chi}$ that entered in the Lagrangian density and their combination $\left(4 \lambda_{\Phi} \lambda_{\chi}-\lambda_{\Phi \chi}^{2}\right)$ as function of $\left(m_{\gamma_{D}}, m_{2}\right)$ in the small mass region up to $5 \mathrm{GeV}$ with fixed values of $g_{D}=0.01$ and $\alpha=0.03$. As one can easily see that $\lambda_{\Phi}$ is not sensitive to these input parameters and very close to its SM value of $m_{1}^{2} / 2 v^{2}=0.13$. We note the following hierarchy $\lambda_{\chi} \ll \lambda_{\Phi \chi} \ll \lambda_{\Phi}$ in this small mass region from the first three plots of this figure. Moreover, the positiveness of the combination $\left(4 \lambda_{\Phi} \lambda_{\chi}-\lambda_{\Phi \chi}^{2}\right)$ in the last plot of this figure implies the scalar potential is bounded from below at tree level.

In figure 3, we plot the trilinear couplings $\lambda_{3}^{(2)} / v, \lambda_{3}^{(3)} / v$ and $\lambda_{3}^{(4)} / v$ normalized to the $\mathrm{VEV} v$, and the quadrilinear coupling $\lambda_{4}^{(3)}$ that are relevant to the three body processes $h_{1} \rightarrow h_{2} \gamma_{D} \gamma_{D}$ and $h_{1} \rightarrow h_{2} h_{2} h_{2}$ as function of $\left(m_{\gamma_{D}}, m_{2}\right)$ in the small mass region up to

\footnotetext{
${ }^{4}$ We would like to thank W. Y. Keung bringing us the attention of this experimental paper.
} 

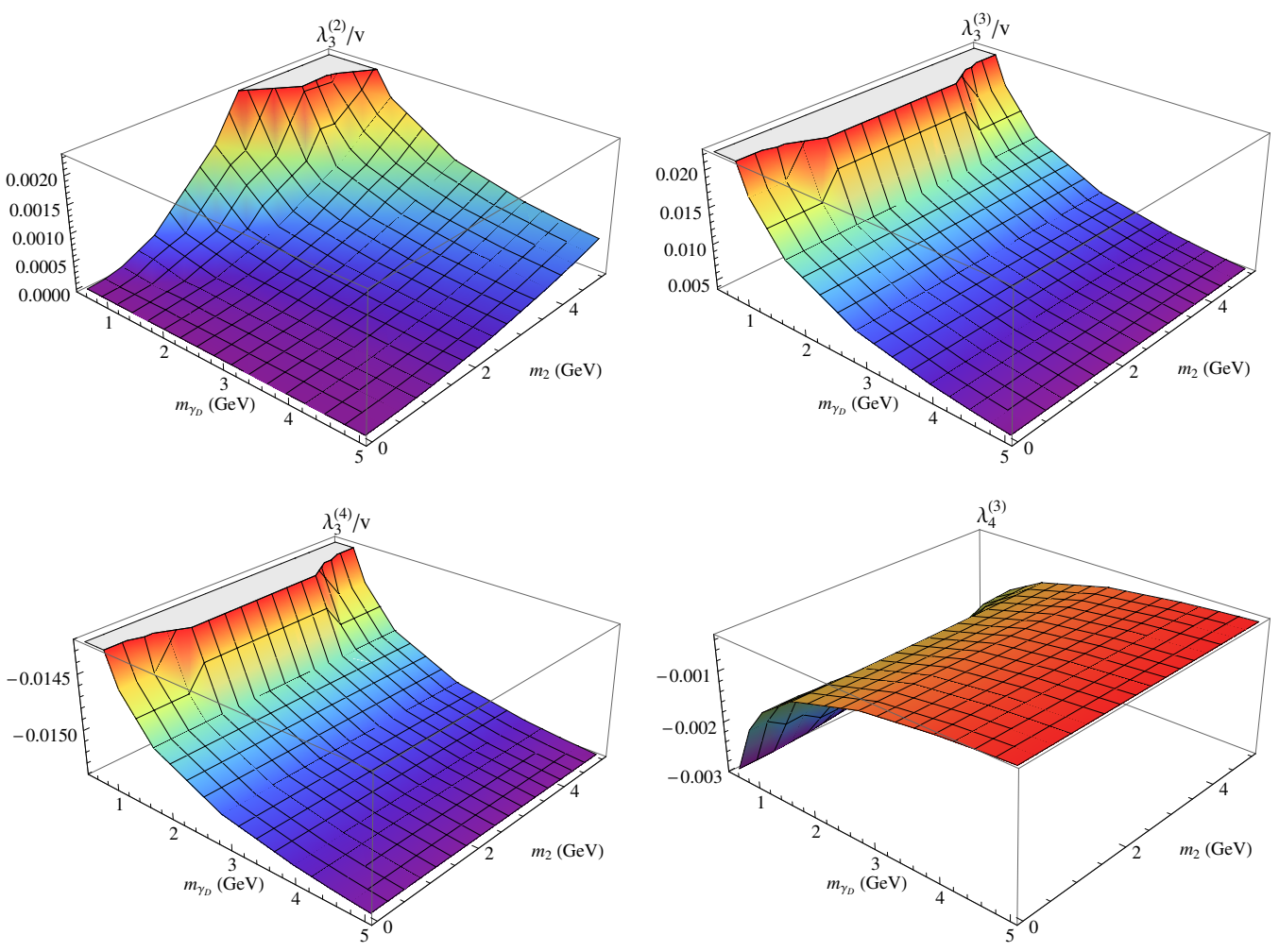

Figure 3. Couplings $\lambda_{3}^{(2)} / v, \lambda_{3}^{(3)} / v, \lambda_{3}^{(4)} / v$ and $\lambda_{4}^{(3)}$ as function of $\left(m_{\gamma_{D}}, m_{2}\right)$ in the small mass region up to $5 \mathrm{GeV}$ with fixed values of $g_{D}=0.01$ and $\alpha=0.03$.

$5 \mathrm{GeV}$ with fixed values of $g_{D}=0.01$ and $\alpha=0.03$. We note that the matrix element (eq. (4.8)) for the three body process $h_{1} \rightarrow h_{2} h_{2} h_{2}$ involves one term proportional to the quartic coupling $\lambda_{4}^{(3)}$ and two other terms proportional to the product of cubic couplings $\lambda_{3}^{(2)} \cdot \lambda_{3}^{(3)}$ and $\lambda_{3}^{(3)} \cdot \lambda_{3}^{(4)}$ respectively, while the matrix element for the three body process $h_{1} \rightarrow h_{2} \gamma_{D} \gamma_{D}$ involves diagrams proportional to $g_{D}^{2} \sin \alpha \cos \alpha, g_{D}^{2} \lambda_{3}^{(3)} \cos \alpha, g_{D}^{2} \lambda_{3}^{(4)} \sin \alpha$ or $g_{D}^{4} \sin \alpha \cos \alpha$. The dark gauge coupling $g_{D}$ alone in general is not too severely constrained by experiments. ${ }^{5}$ On the other hand, since the $126 \mathrm{GeV}$ new boson observed at the LHC behaves very much SM-like, the mixing angle $\alpha$ is constrained to be quite small. Thus the three body decay $h_{1} \rightarrow h_{2} \gamma_{D} \gamma_{D}$ is expected to be more relevant than $h_{1} \rightarrow h_{2} h_{2} h_{2}$. In our analysis, we include both of these three body modes and find that the mode $h_{1} \rightarrow h_{2} h_{2} h_{2}$ is indeed negligible.

In figure 4 , we plot the contour of the non-standard branching ratio $B_{h_{1}}^{\text {NS }}$ (eq. $\left.(4.3)\right)=$ 0.1 (left) and 0.2 (right) of the heavier Higgs $h_{1}$ in the $\left(m_{\gamma_{D}}, m_{2}\right)$ plane up to $126 \mathrm{GeV}$ in both directions with the following parameter input: $\sin ^{2} \alpha=0.0009$ and $g_{D}=0.05,0.1$, $0.2,0.4$ and 0.8 .

In figure 5 , we plot the contour of the non-standard branching ratio $B_{h_{1}}^{\mathrm{NS}}$ (eq. $\left.(4.3)\right)=$ 0.1 (left) and 0.2 (right) of the heavier Higgs $h_{1}$ in the $\left(m_{\gamma_{D}}, m_{2}\right)$ plane for the small mass

\footnotetext{
${ }^{5}$ At the low mass region of the dark photon and dark Higgs that we are interested in, the $B A B A R$ experiment [23] had only obtained the limit for the product $\alpha_{D} \cdot \epsilon^{2}$, where $\alpha_{D}=g_{D}^{2} / 4 \pi$ and $\epsilon$ is the kinetic mixing parameter in eq. (2.2), as a function of the dark Higgs mass or dark photon mass.
} 

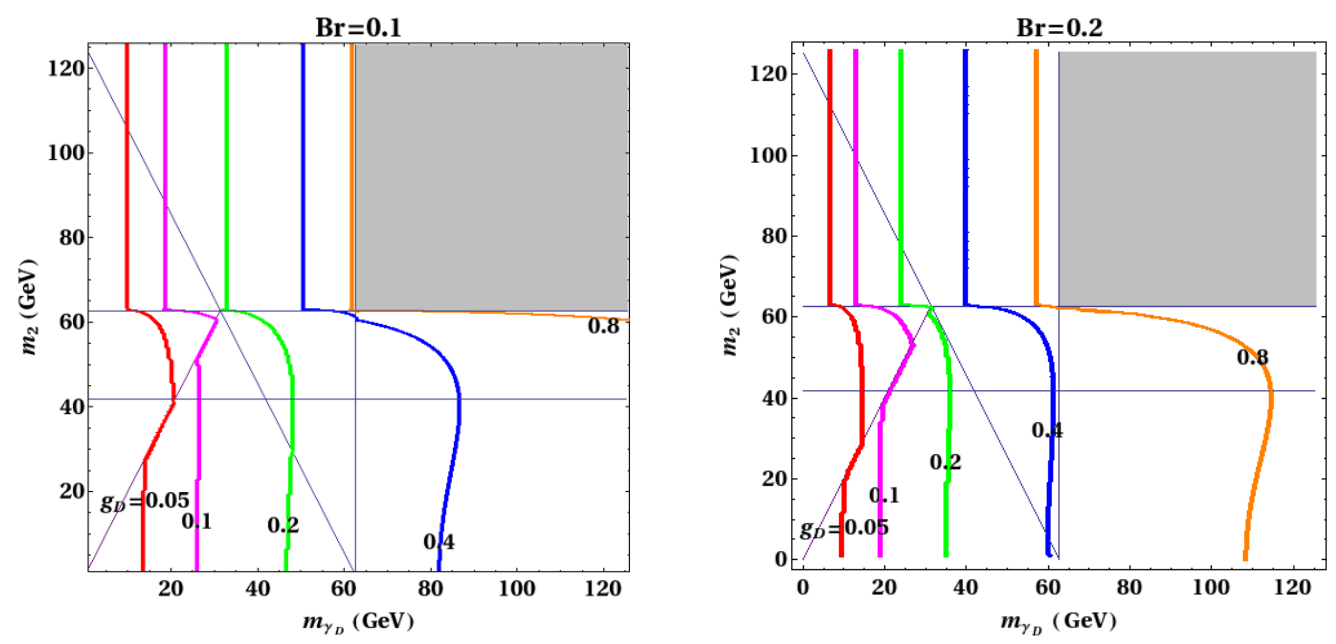

Figure 4. Contour plot of the non-standard branching ratio $B_{h_{1}}^{\mathrm{NS}}$ (eq. (4.3)) $=0.1$ (left) and 0.2 (right) of the heavier Higgs $h_{1}$ in the $\left(m_{\gamma_{D}}, m_{2}\right)$ plane up to $126 \mathrm{GeV}$ in both directions for $\sin ^{2} \alpha=0.0009$ and $g_{D}=0.05,0.1,0.2,0.4$ and 0.8 .
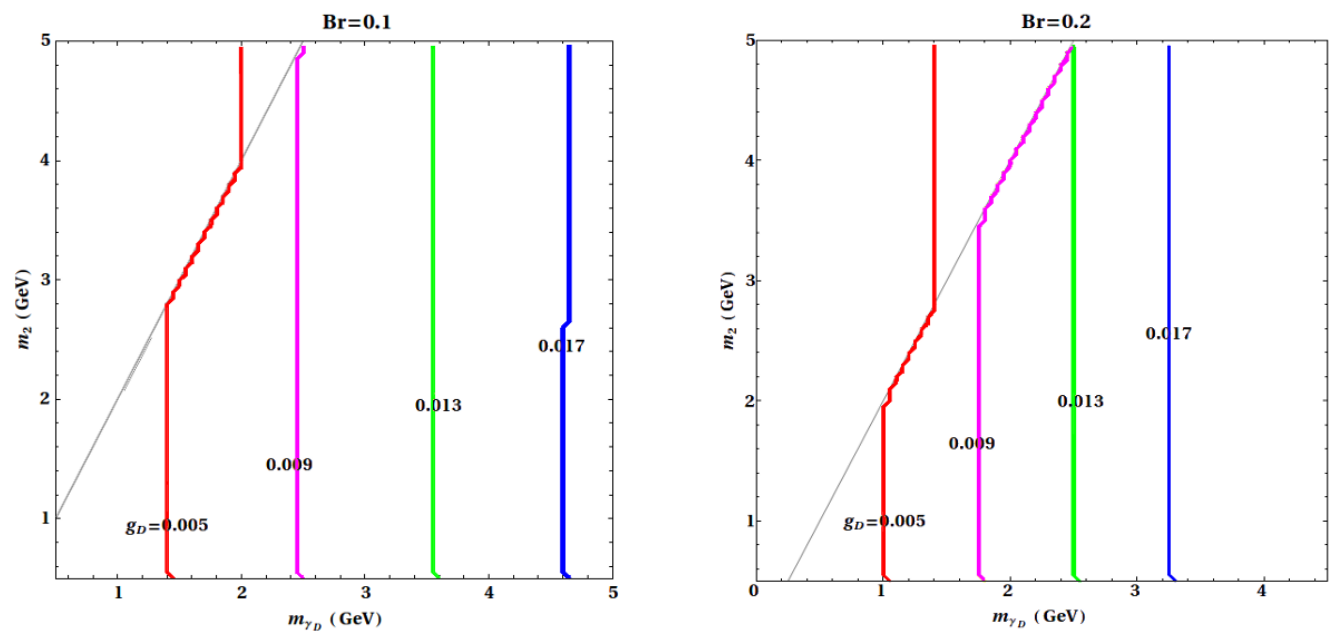

Figure 5. Contour plot of the non-standard branching ratio $B_{h_{1}}^{\mathrm{NS}}$ (eq. $\left.(4.3)\right)=0.1$ (left) and 0.2 (right) of the heavier Higgs $h_{1}$ in the small mass region of 0.5 to $5 \mathrm{GeV}$ in the $\left(m_{\gamma_{D}}, m_{2}\right)$ plane for $\sin ^{2} \alpha=0.0009$ and $g_{D}=0.005,0.009,0.013$ and 0.017 .

region of 0.5 to $5 \mathrm{GeV}$ in both directions for $\sin ^{2} \alpha=0.0009$ and $g_{D}=0.005,0.009,0.013$ and 0.017 .

\section{Multilepton jets at the LHC}

We will study some collider signatures for the model in this section. In particular, we will focus on the 4 lepton-jets and 2 lepton-jets modes in our analysis. We consider the following four processes which may lead to signals of multilepton jets at the LHC:

(I) $p p \rightarrow h \rightarrow Z Z \rightarrow l^{+} l^{-} l^{+} l^{-}$

(II) $p p \rightarrow V V \rightarrow l^{+} l^{-} l^{+} l^{-} \quad(V V=Z Z, \gamma \gamma, Z \gamma)$ 

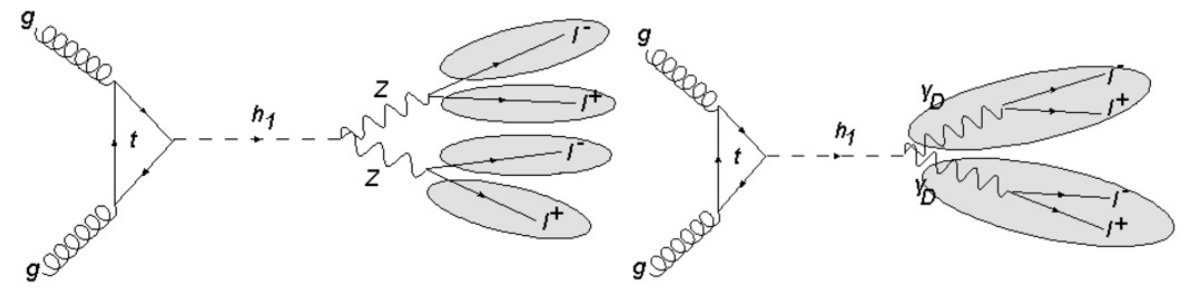

Figure 6. Some topologies of 4 (left) and 2 (right) lepton-jets for process III. The 4 lepton-jets can also be coming from the SM of process I with $h_{1}$ replaced by the SM $h$. The immediate state of $h_{2} h_{2}$ for the 2 lepton-jets is not shown since the branching ratio for $h_{2} \rightarrow l^{+} l^{-}$is very tiny.

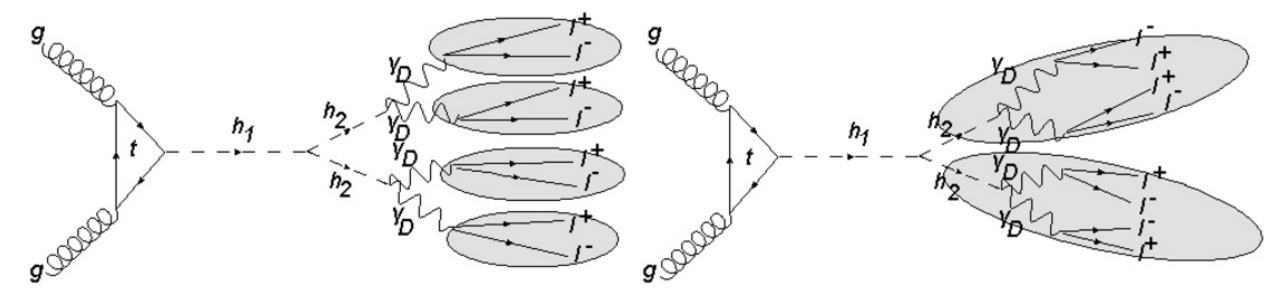

Figure 7. Some topologies of 4 (left) and 2 (right) lepton-jets for process IV.

(III) $p p \rightarrow h_{1} \rightarrow X X \rightarrow l^{+} l^{-} l^{+} l^{-} \quad\left(X X=Z Z, \gamma_{D} \gamma_{D}, h_{2} h_{2}\right)$

(IV) $p p \rightarrow h_{1} \rightarrow h_{2} h_{2} \rightarrow \gamma_{D} \gamma_{D} \gamma_{D} \gamma_{D} \rightarrow l^{+} l^{-} l^{+} l^{-} l^{+} l^{-} l^{+} l^{-}$

where $l=e$ or $\mu$. Processes (I) and (II) are coming entirely from the SM, process (III) can be arise from either SM (with modified Higgs- $Z Z$ coupling) or the dark portal (see figure 6), and process (IV) is purely from the dark portal (see figure 7).

We compute the matrix elements of these processes using FeynRules ${ }^{6}[24,25]$ and MadGraph [26]. We pass these matrix elements to the event generator MadEvent [27] to obtain our event samples. The set of parton distribution functions used is CTEQ6L1 [28].

For illustration, we will choose several benchmark points in the dark portal as shown in table 1. If the kinetic mixing parameter $\epsilon$ is smaller than $10^{-5}$, the dark photon will have a very long lifetime and it may decay outside the detector. We will choose it to be $10^{-4}$ as used by previous analyses by theorists [12] as well as experimentalists [29]. The mass of dark photon is chosen to be less than $2 \mathrm{GeV}$ in these benchmark points. With such relatively low mass the opening angle of the lepton pair from the decay of the dark photon will be small which may lead to multilepton jets. Such low mass dark photon may also be desirable for indirect dark matter searches, since the allowed decay $\gamma_{D} \rightarrow e^{+} e^{-}$may be used to explain the positron excess [30-32], while $\gamma_{D} \rightarrow p \bar{p}$ is kinematically disallowed in accord with observation that the cosmic anti-proton flux is consistent with the background [30,33]. We also choose $\sin ^{2} \alpha=10^{-3}$ in consistent with the analysis of the invisible branching ratio of $h_{1}$ in previous section (see also [34] and [35, 36]). At these benchmark points, we see from the last three columns of table 1 that (1) the invisible decay branching ratio of the SM Higgs is consistent with global fit results, (2) the decay of the dark Higgs is almost $100 \%$

\footnotetext{
${ }^{6}$ We include both gluon and photon fusion $g g \rightarrow h_{1}$ and $\gamma \gamma \rightarrow h_{1}$ computed at next-to-leading-order.
} 


\begin{tabular}{|ccccccc|}
\hline Benchmark Point & $g_{D}$ & $M_{\gamma_{D}}$ & $m_{2}$ & $\mathrm{Br}_{h_{1} \rightarrow \text { DarkStuff }}$ & $\mathrm{Br}_{h_{2} \rightarrow \gamma_{D} \gamma_{D}}$ & $\mathrm{Br}_{\gamma_{D} \rightarrow l^{+} l^{-}}$ \\
\hline A & 0.005 & 1.5 & 4 & $\sim 16 \%$ & $99 \%$ & $50 \%$ \\
B & 0.009 & 1.8 & 10 & $\sim 20 \%$ & $100 \%$ & $50 \%$ \\
C & 0.005 & 1.5 & 40 & $\sim 15 \%$ & $99 \%$ & $50 \%$ \\
D & 0.005 & 1.8 & 40 & $\sim 11 \%$ & $99 \%$ & $50 \%$ \\
\hline
\end{tabular}

Table 1. Several benchmark points of the dark portal used to calculate the signals of multilepton jets $\left(\epsilon=10^{-4}\right.$ and $\left.\sin ^{2} \alpha=10^{-3}\right)$.

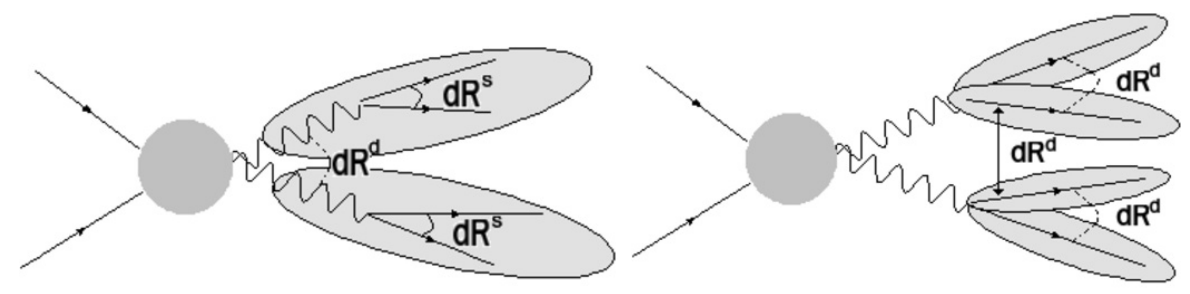

Figure 8. Graphical illustrations for the kinematic cuts on the cone radius $\Delta R$ of final state leptons. The 2 and 4 lepton-jets cases are shown in the left and right figures respectively.

into pair of dark photons, and (3) the branching ratio of the dark photon into light lepton pairs can be as large as 50\%. Due to the smallness of the two mixing parameters $\alpha$ and $\epsilon$, the production cross section of $h_{1}$ at the LHC remains to be very close to its SM value.

For the kinematic cuts for the 2 and 4 lepton-jets, we follow refs. [9, 12] and [29]. For the basic cuts that we will impose in all processes, we have

$$
\begin{array}{llll}
\text { Basic cuts: } & \text { (4 leptons case) } & p_{T_{l}} \geq 20,10,10,10 \mathrm{GeV}, & \left|\eta_{l}\right|<2.3 ; \\
& \text { (8 leptons case) } & p_{T_{l}} \geq 20,10,10,10,0,0,0,0 \mathrm{GeV}, & \left|\eta_{l}\right|<2.3,
\end{array}
$$

where $p_{T_{l}}$ and $\eta_{l}$ are the transverse momenta and pseudo-rapidity of the lepton respectively. On top of the basic cuts, we employ the following lepton-jets cuts

$$
\begin{array}{llll}
4 \text { lepton-jets cuts : } & \Delta R_{j_{i} j_{j}}^{d}>0.7, & \Delta R_{l_{i} l_{j}}^{s}<0.2, & M_{\text {Invariant }}=M_{h_{1}} \pm 10 \mathrm{GeV} ; \\
2 \text { lepton-jets cuts : } & \Delta R_{j_{1} j_{2}}^{d}>0.7, & \Delta R_{l_{i} l_{j}}^{s}<0.2, & M_{\text {Invariant }}=M_{h_{1}} \pm 10 \mathrm{GeV} .
\end{array}
$$

Here $\Delta R_{j j}^{d}$ denotes the cone radius between two different lepton-jets and $\Delta R_{l l}^{s}$ denotes the cone radius between two different leptons in the same lepton jet, as depicted in figure 8 . $M_{\text {Invariant }}$ denotes the invariant mass of all final state particles due to the decay chain of the SM Higgs boson resonance, give or take $10 \mathrm{GeV}$ from the central value of $126 \mathrm{GeV}$.

The number of events versus the total invariant mass $M_{\text {Invariant }}$ for the four processes I, II, III and IV at the LHC-14 without any cuts are shown in figure 9 for the benchmark point B. We can see that before imposing any cuts the number of events around the Higgs boson resonance for the two processes III (red) and IV (yellow, 8 leptons) from the dark portal can stand above the SM processes of I (blue) and II (black). However away from the resonance region, the 4 leptons SM background from process I (black) is 2 to 3 order of magnitudes above the signals from process IV (green). 


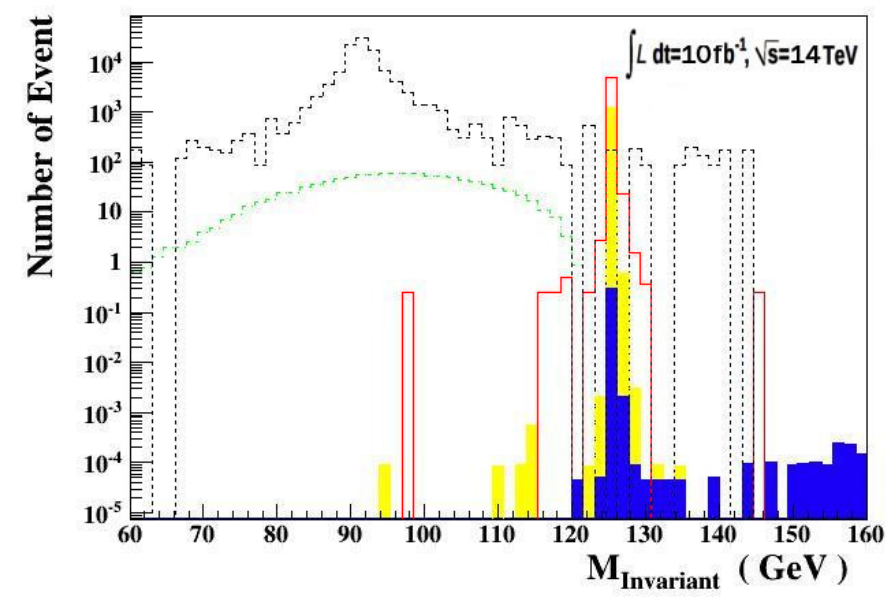

Figure 9. Number of events versus $M_{\text {Invariant }}[\mathrm{GeV}]$ with the basic cuts for benchmark point B at LHC-14 with a fixed luminosity of $10 \mathrm{fb}^{-1}$. Histogram of blue strip is for process I, black dash is for process II, red solid is for process III, yellow strip is for process IV of 8 leptons, and green dash is for process IV of 4 leptons.

We now discuss the impact of imposing the multilepton jets cuts on the cross sections. The topologies of imposing the 4 and 2 lepton-jets cuts for processes III and IV are shown in figures 6 and 7 respectively. In table 2, we show the cross sections of the 4 processes at the LHC-14 with the basic, 4 and 2 lepton-jets cuts for the 4 benchmark points listed in table 1 . The following statements can be drawn from the results shown in table 2 :

- The 4 and 2 lepton jets cuts have strong and different impact for the SM processes I and II. For process I, since the intermediate state is the $Z$ boson with a relatively high mass, its decay products can be produced at a relatively large angle with respect to the original $Z$ boson direction. Thus it favors 4 lepton-jets in the final state (see left diagram in figure 6) and 2 lepton-jets is vanishing small for process I. On the other hand, SM process II has a cross section of about 700 times larger than process I with just the basic cuts imposed. Imposing the 4 and 2 lepton-jets cuts reduce the cross section of process II by a factor of $4.7 \times 10^{-3}$ and $1.1 \times 10^{-3}$ respectively. We note that the $Z Z$ intermediate state in process II arises from the tree level parton processes of quark-quark annihilation while in process I it is connected with the loop-induced gluon fusion mechanism of Higgs production.

- For process III since the dark photon mass is small $(1.5 \mathrm{GeV}$ for benchmark points $\mathrm{A}$ and $\mathrm{C}$, and $1.8 \mathrm{GeV}$ for benchmark points $\mathrm{B}$ and $\mathrm{D})$ the contribution from intermediate state of $\gamma_{D} \gamma_{D}$ will give rise mainly to 2 lepton jets (see right diagram in figure 6). Thus imposing the 4 lepton jets cuts for process III will suppress this intermediate state and only the contribution from $Z Z$ intermediate state will survive (see left diagram in figure 6). Since this $Z Z$ contribution is very similar to the SM process I, they should have very similar cross sections after imposing 4 lepton-jets cuts as clearly seen in table 2 . On the other hand, imposing 2 lepton-jets cuts will suppress the $Z Z$ intermediate state but keep the $\gamma_{D} \gamma_{D}$. However, the contribution 


\begin{tabular}{|lccccr|}
\hline Cuts & Benchmark Point & I & II & III & \multicolumn{1}{c|}{ IV } \\
\hline Basic & A & 0.118 & 70.7 & 95.3 & 23.2 \\
& B & 0.118 & 70.7 & 204 & 45.8 \\
& C & 0.118 & 70.7 & 96.7 & 19.2 \\
& D & 0.118 & 70.7 & 68.3 & 13.1 \\
\hline Basic + 4 Lepton-Jets & A & $9.63 \times 10^{-3}$ & 0.337 & $9.86 \times 10^{-3}$ & $\leq 10^{-10}$ \\
& B & $9.63 \times 10^{-3}$ & 0.337 & $9.80 \times 10^{-3}$ & $\leq 10^{-10}$ \\
& C & $9.63 \times 10^{-3}$ & 0.337 & $9.93 \times 10^{-3}$ & 3.05 \\
& D & $9.63 \times 10^{-3}$ & 0.337 & $9.84 \times 10^{-3}$ & 0.92 \\
\hline Basic + 2 Lepton-Jets & A & $\leq 10^{-10}$ & 0.08 & 95.3 & 1.75 \\
& B & $\leq 10^{-10}$ & 0.08 & 201 & $\leq 10^{-10}$ \\
& C & $\leq 10^{-10}$ & 0.08 & 95.8 & $\leq 10^{-10}$ \\
& D & $\leq 10^{-10}$ & 0.08 & 68.2 & $\leq 10^{-10}$ \\
\hline
\end{tabular}

Table 2. Cross sections (in unit of $\mathrm{fb}$ ) at the LHC-14 for the background processes (I and II) and dark sector processes (III and IV) with the basic, 4 and 2 lepton-jets cuts at the 4 benchmark points.

of $Z Z$ intermediate state for process III is negligible. The 2 lepton-jets cross sections of process III are several orders of magnitudes larger than the corresponding cross sections of SM process II.

- For process IV, with just basic cuts its cross section is about a factor 4 (benchmark points $\mathrm{A}$ and $\mathrm{B}$ ) to 5 (benchmark points $\mathrm{C}$ and $\mathrm{D}$ ) smaller than that of process III. However, due to the small mass of the dark photon (compared with $Z$ boson mass), one can has either 4 or 2 lepton-jets in the final state. Imposing the 4 and 2 lepton-jets cuts in addition to the basic cuts for process IV have more nontrivial effects on the cross section depending on the benchmark points. For 4 lepton-jets the cross sections can reach about 3 and 1 femtobarn for benchmark points $\mathrm{C}$ and $\mathrm{D}$ respectively. For 2 lepton-jets, the cross section can reach 2 femtobarn for benchmark point A only. At these benchmark points, these cross sections are an order of magnitude larger than the corresponding cross sections of the SM process II. Other benchmark points have negligible cross sections for 4 and 2 lepton-jets as can be clearly seen in the last column of table 2 .

\section{Conclusions}

We have studied a simple extension of the SM by adding a dark sector described by the original Abelian Higgs model. The communication between the visible sector and the dark sector is due to the mixing between the SM and dark Higgses and/or mixing between the SM and dark photons. We study various non-standard decay modes of the heavier Higgs $h_{1}$ in this model, identified as the $126 \mathrm{GeV}$ new boson observed recently at the LHC. Multilepton modes in the final states of this heavier Higgs decay are possible. For the case 
of $h_{1} \rightarrow h_{2} h_{2}$ followed by $h_{2} \rightarrow \gamma_{D} \gamma_{D}$ and $\gamma_{D} \rightarrow \overline{l l}$, there could be eight leptons in the final states. The three body process $h_{1} \rightarrow h_{2} \gamma_{D} \gamma_{D}$ is found to be significant and could lead to eight leptons final state as well. On the other hand, the other three body process $h_{1} \rightarrow h_{2} h_{2} h_{2}$ has an insignificant branching ratio; otherwise, it would lead up to a even more spectacular twelve leptons final state. The signals of 4 and 2 lepton-jets in this model are already quite unique and spectacular. We show that there are parameter space in this simple dark portal model satisfying the current constraint of the non-standard decay width of the $126 \mathrm{GeV}$ Higgs and may give rise to interesting signals of multilepton jets at the LHC-14. Experiments at the LHC should therefore search for multilepton modes in the Higgs decay in order to probe for the possible existence of a $\mathrm{U}(1)_{D}$ dark sector governed by the original Abelian Higgs model.

\section{Acknowledgments}

EM would like to thank the hospitality of the Institute of Physics, Academia Sinica, Taipei, Taiwan. We would like to thank Chuan-Ren Chen, Sunghoon Jung, Zuowei Liu and Olivier Mattelaer for many useful communications. This work is supported in part by the U. S. Department of Energy under Grant No. DE-SC0008541 and by National Science Council of Taiwan under grant 101-2112-M-001-005-MY3.

Open Access. This article is distributed under the terms of the Creative Commons Attribution License (CC-BY 4.0), which permits any use, distribution and reproduction in any medium, provided the original author(s) and source are credited.

\section{References}

[1] P.W. Higgs, Broken symmetries and the masses of gauge bosons, Phys. Rev. Lett. 13 (1964) 508 [INSPIRE].

[2] F. Englert and R. Brout, Broken symmetry and the mass of gauge vector mesons, Phys. Rev. Lett. 13 (1964) 321 [INSPIRE].

[3] G.S. Guralnik, C.R. Hagen and T.W.B. Kibble, Global conservation laws and massless particles, Phys. Rev. Lett. 13 (1964) 585 [INSPIRE].

[4] S. Weinberg, A model of leptons, Phys. Rev. Lett. 19 (1967) 1264 [InSPIRE].

[5] ATLAS collaboration, Observation of a new particle in the search for the standard model Higgs boson with the ATLAS detector at the LHC, Phys. Lett. B 716 (2012) 1 [arXiv: 1207.7214] [INSPIRE].

[6] CMS collaboration, Observation of a new boson at a mass of $125 \mathrm{GeV}$ with the CMS experiment at the LHC, Phys. Lett. B 716 (2012) 30 [arXiv:1207.7235] [INSPIRE].

[7] B. Holdom, Two U(1)'s and $\epsilon$ charge shifts, Phys. Lett. B 166 (1986) 196 [InSPIRE].

[8] B. Holdom, Searching for $\epsilon$ charges and a new U(1), Phys. Lett. B 178 (1986) 65 [INSPIRE].

[9] S. Gopalakrishna, S. Jung and J.D. Wells, Higgs boson decays to four fermions through an abelian hidden sector, Phys. Rev. D 78 (2008) 055002 [arXiv:0801.3456] [INSPIRE]. 
[10] H. Davoudiasl, H.-S. Lee, I. Lewis and W.J. Marciano, Higgs decays as a window into the dark sector, Phys. Rev. D 88 (2013) 015022 [arXiv:1304.4935] [INSPIRE].

[11] H. Davoudiasl, H.-S. Lee and W.J. Marciano, 'Dark' Z implications for parity violation, rare meson decays and Higgs physics, Phys. Rev. D 85 (2012) 115019 [arXiv:1203.2947] [INSPIRE].

[12] A. Falkowski, J.T. Ruderman, T. Volansky and J. Zupan, Discovering Higgs decays to lepton jets at hadron colliders, Phys. Rev. Lett. 105 (2010) 241801 [arXiv:1007.3496] [INSPIRE].

[13] A. Falkowski, J.T. Ruderman, T. Volansky and J. Zupan, Hidden Higgs decaying to lepton jets, JHEP 05 (2010) 077 [arXiv: 1002.2952] [INSPIRE].

[14] D. Feldman, Z. Liu and P. Nath, The Stückelberg Z-prime extension with kinetic mixing and milli-charged dark matter from the hidden sector, Phys. Rev. D 75 (2007) 115001 [hep-ph/0702123] [INSPIRE].

[15] K. Cheung, J.S. Lee and P.-Y. Tseng, Higgs precision (Higgcision) era begins, JHEP 05 (2013) 134 [arXiv:1302.3794] [inSPIRE].

[16] P.P. Giardino, K. Kannike, I. Masina, M. Raidal and A. Strumia, The universal Higgs fit, arXiv: 1303.3570 [INSPIRE].

[17] J. Ellis and T. You, Updated global analysis of Higgs couplings, JHEP 06 (2013) 103 [arXiv:1303.3879] [INSPIRE].

[18] G. Bélanger, B. Dumont, U. Ellwanger, J. Gunion and S. Kraml, Global fit to Higgs signal strengths and couplings and implications for extended Higgs sectors, Phys. Rev. D 88 (2013) 075008 [arXiv: 1306.2941] [INSPIRE].

[19] E.C.G. Stückelberg, Die Wechselwirkungskräfte in der Elektrodynamik und in der Feldtheorie der Kräfte, Helv. Phys. Acta 11 (1938) 225.

[20] V.I. Ogievetskii and I.V. Polubarinov, A gauge invariant formulation of neutral vector field theory, Sov. Phys. JETP 14 (1962) 179.

[21] CMS collaboration, Search for anomalous production of multilepton events in pp collisions at $\sqrt{s}=7 \mathrm{TeV}, J H E P O 6$ (2012) 169 [arXiv:1204.5341] [INSPIRE].

[22] OPAL collaboration, G. Abbiendi et al., Search for invisibly decaying Higgs bosons in

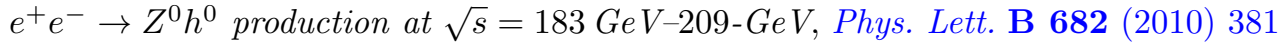
[arXiv: 0707.0373] [INSPIRE].

[23] BaBAr collaboration, J. Lees et al., Search for low-mass dark-sector Higgs bosons, Phys. Rev. Lett. 108 (2012) 211801 [arXiv:1202.1313] [INSPIRE].

[24] N.D. Christensen and C. Duhr, FeynRules - Feynman rules made easy, Comput. Phys. Commun. 180 (2009) 1614 [arXiv:0806.4194] [INSPIRE].

[25] A. Alloul, B. Fuks and V. Sanz, Phenomenology of the Higgs effective lagrangian via FeynRules, arXiv:1310.5150 [INSPIRE].

[26] J. Alwall, M. Herquet, F. Maltoni, O. Mattelaer and T. Stelzer, MadGraph 5: going beyond, JHEP 06 (2011) 128 [arXiv:1106.0522] [INSPIRE].

[27] F. Maltoni and T. Stelzer, MadEvent: automatic event generation with MadGraph, JHEP 02 (2003) 027 [hep-ph/0208156] [INSPIRE].

[28] J. Pumplin, A. Belyaev, J. Huston, D. Stump and W.K. Tung, Parton distributions and the strong coupling: CTEQ6AB PDFs, JHEP 02 (2006) 032 [hep-ph/0512167] [INSPIRE]. 
[29] ATLAS collaboration, Search for WH production with a light Higgs boson decaying to prompt electron-jets in proton-proton collisions at $\sqrt{s}=7 \mathrm{TeV}$ with the ATLAS detector, New J. Phys. 15 (2013) 043009 [arXiv:1302.4403] [InSPIRE].

[30] PAMELA collaboration, O. Adriani et al., An anomalous positron abundance in cosmic rays with energies 1.5-100 GeV, Nature 458 (2009) 607 [arXiv:0810.4995] [INSPIRE].

[31] Fermi LAT collaboration, A.A. Abdo et al., Measurement of the cosmic ray $e^{+}$plus $e^{-}$ spectrum from $20 \mathrm{GeV}$ to $1 \mathrm{TeV}$ with the Fermi Large Area Telescope, Phys. Rev. Lett. 102 (2009) 181101 [arXiv:0905.0025] [INSPIRE].

[32] AMS collaboration, M. Aguilar et al., First result from the Alpha Magnetic Spectrometer on the International Space Station: precision measurement of the positron fraction in primary cosmic rays of 0.5-350 GeV, Phys. Rev. Lett. 110 (2013) 141102 [INSPIRE].

[33] PAMELA collaboration, O. Adriani et al., PAMELA results on the cosmic-ray antiproton flux from $60 \mathrm{MeV}$ to $180 \mathrm{GeV}$ in kinetic energy, Phys. Rev. Lett. 105 (2010) 121101 [arXiv: 1007.0821] [INSPIRE].

[34] J.D. Clarke, R. Foot and R.R. Volkas, Phenomenology of a very light scalar (100 MeV $<m_{h}<10 \mathrm{GeV}$ ) mixing with the SM Higgs, JHEP 02 (2014) 123 [arXiv:1310.8042] [INSPIRE].

[35] L3 collaboration, M. Acciarri et al., Search for neutral Higgs boson production through the process $e^{+} e^{-} \rightarrow Z^{*} H^{0}$, Phys. Lett. B 385 (1996) 454 [InSPIRE].

[36] LEP Higgs Working for Higgs boson searches, ALEPH, DELPHI, CERN-L3, OPAL collaboration, Searches for invisible Higgs bosons: preliminary combined results using LEP data collected at energies up to $209 \mathrm{GeV}$, hep-ex/0107032 [INSPIRE]. 